\title{
ADIESTRAMIENTO ETOLÓGICO DEL CABALLO
}

\author{
Ethological training of the horse
}

\section{Artículo de Investigación}

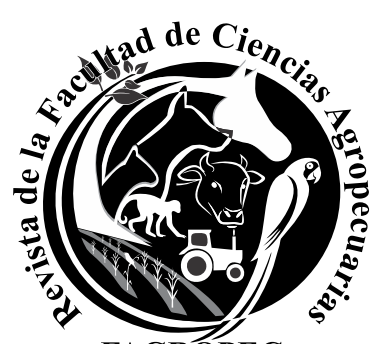

FAGROPEC

Recibido 21 de agosto de 2019. Aceptado 29 de noviembre de 2019.

${ }^{1}$ Docente tiempo completo, Universidad Francisco de Paula Santander, Grupo de investigación GI@DSy GIPAB.

\section{(iD https://orcid.org/0000-0002-0377-4664}

${ }^{2}$ Docente tiempo completo, Universidad Francisco de Paula Santander, Grupo de investigación GI@DS y GIPAB.

iD https://orcid.org/0000-0003-1971-8365

${ }^{3}$ Fundación de Estudios Superiores COMFANORTE, Grupo de investigación GRINFESC y GIPAB.

D https://orcid.org/0000-0001-7718-853X

${ }^{4}$ Docente Universidad de Pamplona, Facultad de Ciencias Agrarias

D https://orcid.org/0000-0003-4035-720X

\section{Como citar:}

HOYOS PATIÑO, J. F., et al. Adiestramiento etológico del caballo. En: Revista Facultad Ciencias Agropecuarias - FAGROPEC. Universidad de la Amazonia, Florencia Caquetá. Volumen 11 julio-diciembre, 2019. Pp. 79-89 ISSN-Revista en Línea: 2539-178X

*Autor para correspondencia: jfhoyosp@ufpso.edu.co
Johann Fernando Hoyos Patiño ${ }^{1^{*}}$ MSc, Daniel Antonio Hernández Villamizar $^{2}$ MSc, Blanca Liliana Velásquez Carrascal $^{3}$ MSc y Eliecer $^{2}$ Franco Roa ${ }^{4}$

\section{RESUMEN}

La doma es pilar fundamental en el proceso productivo de los criaderos y gremio equino. Investigaciones sobre el tema demuestran su relación con estado físico y psicológico del caballo. En este contexto, surgen diferentes tendencias de doma que muestran innumerables alternativas desde las confesionales o tradicionales a las etologías y sin violencia, todas propendiendo desde su ámbito el logro de animales domados, atentos y presto al trabajo. Desde este enfoque, el presente artículo describe aspectos importantes con el fin de propiciar en el lector una postura crítica que sirva para ampliar, a futuro, las alternativas para el proceso de doma del caballo.

Palabras claves:

Doma; Adiestramiento; Caballo; Potro; Etológico.

\section{ABSTRACT}

Dressage is a fundamental pillar in the production process of horse breeders and guilds. Research on the subject shows its relationship with the physical and psychological state of the horse. In this context, there are different tendencies of taming that show innumerable alternatives from the confessional or traditional to the ethologies and without violence, all tending from their scope to achieve domesticated animals, attentive and ready to work. From this approach, the present article describes important aspects in order to encourage the reader to adopt a critical stance that will serve to broaden, in the future, the alternatives for the process of taming the horse.

Key words:

Taming; Training; Horse; Foal; Ethology. 


\section{INTRODUCCIÓN}

El uso del caballo como medio de transporte, militar y deportivo es conocido y desarrollado desde tiempos remotos (Corredor, 2009; De Souza, et al, 2013), pero desde finales del siglo XX el hombre ha encomendado al caballo nuevas tareas como la de servir de apoyo en tratamiento de alteraciones sicológicas y físicas (Calmo, 2015; León y Contreras, 2016; Sanna, 2017).

El Caballo Criollo Colombiano, se caracteriza por su sensibilidad, brío, velocidad y suavidad; poseedor de un fenotipo único, elegante, noble, con movimientos bien definidos, que le permite a su montador gozar de gran quietud durante la monta; dichos rasgos son obtenidos no sólo por una adecuada dotación genética, sino además por un oportuno y acertado proceso de adiestramiento (Estrada, R. 1991; Hernández, 2015; Hoyos y Franco, 2017).

Algunas metodologías de doma usan formas impositivas que atentan contra el bienestar del animal y la seguridad del chalan; y otras que obtiene el máximo de las potencialidades del potro basadas en la etológica (Pisa, et al, 2019; Hoyos et al, 2019; Hoyos et al, 2020). En la actualidad se identifican dos tipos de doma: la tradicional, identificada por imposición, bajo grado de especialidad o profundidad del domador y desarrollada con el uso del botalón y la humanitaria, cimentada en el comportamiento y amplia, capacitación del domador y desarrollada principalmente en el picadero (Martínez, 2016; Patiño y Gómez, 2016; Santos, 2019).

El País los principios de la etológica se aplican en la doma desde hace algún tiempo, con el uso de técnicas fundamentadas en la paciencia y observación del comportamiento del potro y el nivel de aceptación y asimilación del proceso (Hoyos y Hernández, 2020; Hoyos, 2007a). La doma equina vista desde la etología, es el compendio de saberes de la doma tradicional que produce caballos con excelente rienda (Estrada, 1994), conjugados con técnicas modernos de doma y adiestramiento que agilizan la aceptación del equino a la manipulación y contacto con el humano, favoreciendo el desarrollo físico sin afectar la salud del equino. (Costa, et al, 2016; Hoyos, 2008a; Hoyos et al, 2020).

\section{METODOLOGÍA}

Desde el punto de vista del planteamiento metodológico, la presente investigación es de tipo documental; elaborando el estado del arte con el análisis de artículos sobre el proceso de doma del caballo criollo; lo anterior enfocado en la perspectiva hermenéutica. La ruta metodológica desarrollada comprendido tres fases: exploración, selección y profundización.

\section{Desarrollo del tema}

La doma, como interacción del hombre con el animal (potro), tiene como finalidad la obtención de un caballo que obedezca órdenes específicas, para el cumplimiento de una tarea puntual (Vieira, 2015). Al momento de acelerar dicho proceso usando la imposición, se disminuye la capacidad del caballo para asimilar la nueva información, activando sus instintos de defensa y huida (Hoyos, 2009a), elevando los niveles de estrés expresados en el aumento de la frecuencia cardiaca, frecuencia respiratoria y niveles de la glucosa en sangre (Martos y Ayala, 2003), llegando a comprometer la sobrevivencia del animal. 
El proceso de doma del potro, inicia con el Imprinting o estimulación temprana, tomando como punto de partida etológico el "período sensible", comprendido desde el nacimiento hasta el segundo día de vida, en donde el potro por observación recibe de la yegua información necesaria sobre la manada, lenguaje gestual, jerárquica, mecanismos básicos huida (Hoyos, 2006; Hoyos, 2007b).

Este proceso se aplica hasta el décimo día (Figura 1 y 2), utilizando la manipulación y el contacto del potro con todos los estímulos existentes en su entorno; va desde la sensibilización al contacto del humano por todo el cuerpo y la relación con herramientas que serán usadas en el futuro proceso de doma. Al igual, que las primera cabestreada, cepillado y sujeción a un botalón (Hoyos, 2007c; Oliveira, et al, 2016).
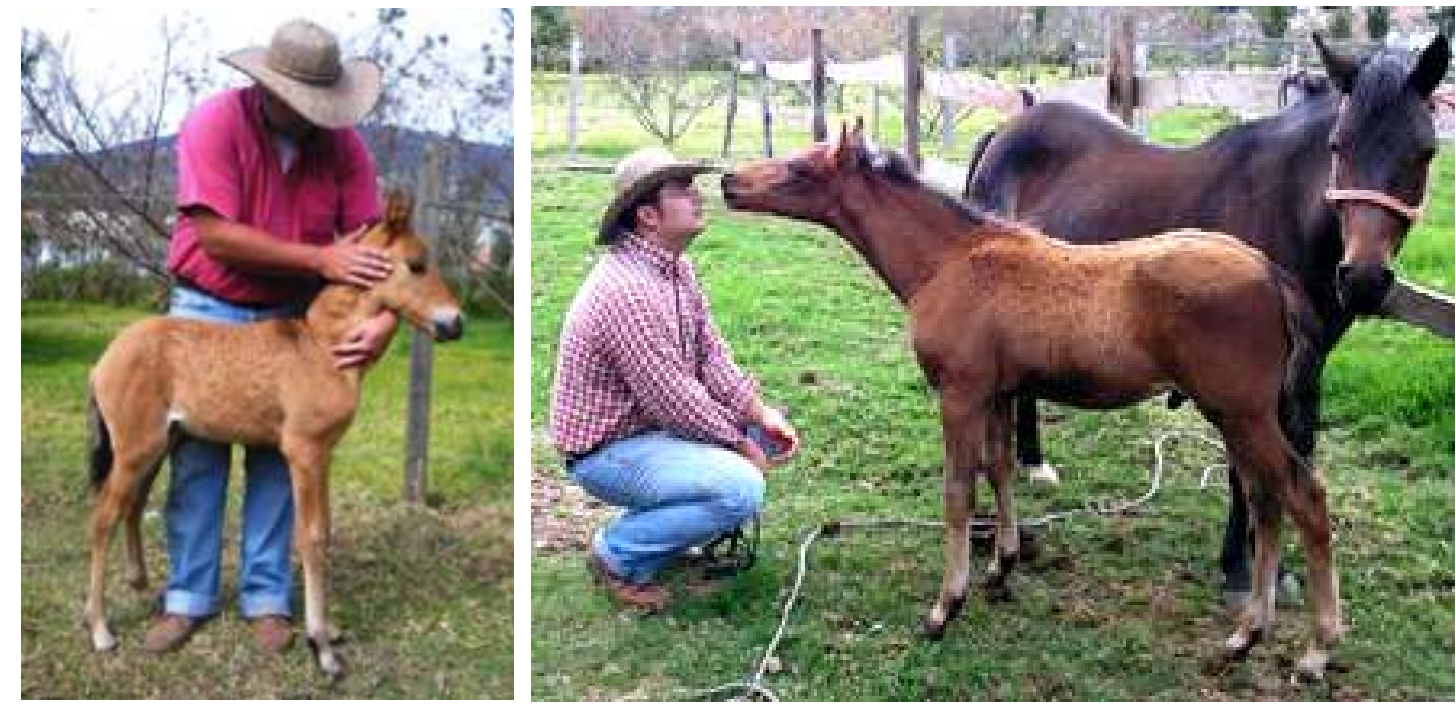

Figura 1 y 2. Potros Improntados. Fuente: Revista Universo Equino No2

Con la aplicación del Imprinting se obtienen potros dóciles y relajados, en comparación con potros que tengan nula manipulación hasta el destete, estos se muestran temerosos y asustadizos a la manipulación humana, complicando así todas las labores de manejo, todo esto se manifiesta a la edad de doma donde los potros improntados, responden a los estímulos de forma tranquila y atenta (Hoyos-Patiño, 2016; Reinos \& Patiño, 2017; Oliveira \& Fergitz, (2018).

Con el fin de aprovechar al máximo el período entre los 7 a los 18 meses de edad del potro, se puede aplicar la Doma de Pesebrera, que tiene como propósitos principales afianzar la habituación hecha en el Imprinting y reafirmar docilidad y tranquilidad; que cabestree, entregue patas y manos, permanezca tranquilamente amarrado de un botalón, acepte la administración de medicamentos por vía oral, entre otras actividades típicas del manejo cotidiano en un criadero o pesebrera (Hoyos, 2008b).

Se busca ganar la confianza del potro, logrando aceptación dentro de la zona de protección (perímetro imaginario del caballo, dentro del cual se puede sentir seguro y tranquilo ante la presencia de algún estímulo nuevo (Hempfling, 1996). Cabe resaltar, que todas estas técnicas de habituación se basan en la caricia, permitiendo siempre que el potro olfatee y reconozca previamente (Hoyos, 2007d). 
Cuando se tiene contacto con potros de 10 meses en adelante se inicia el trabajar dentro de un picadero de una forma suave, introduciéndolos en un trabajo de acondicionamiento físico inicial para preparar la doma, realizando el trabajo al paso para no forzar las articulaciones (Hoyos, 2008c).

Dentro de la herramientas usadas para el manejo del potro y realizar sus primeros movimientos ya sea cabestreado o dentro del picadero, el uso del cabezal de nudos; este se convierte en el punto de unión entre el Hombre y el Caballo, transmitiendo una serie de mandos que condicionan al potro a obedecer órdenes, van acompañados de mandos de voz para reafirmar el condicionamiento. La cabeza del caballo presenta 3 puntos donde se concentran los impulsos que envía el domador a través del cabezal: la nuca, hueso nasal (nariz) y mandíbula (barbilla) (Hoyos, 2008d).

El caballo responderá correctamente a las ayudas y rutinas de entrenamiento, si se encuentra a gusto con el domador y con el medio que lo rodea, esto se logra supliendo sus necesidades sociales, físicas y psíquicas. En muchos casos el domador quiere brindar espacios pero no es posible, dando como resultado la aparición de vicios como aerofagia y manifestaciones de agresividad, inseguridad, irregularidades en la marcha (Quiroz, et al, 2015a). En ocasiones, la forma de reaccionar del humano es la violencia, con la cual se reafirma, fortalece y perpetúa el vicio (Hempfling, 1996; Hoyos 2009b).

Para suplir estas necesidades se deben tener conocimientos básicos sobre los comportamientos típicos del caballo para conseguir una habituación progresiva dando mejores resultados. Los pilares en los cuales se basa el proceso deben ser: paciencia, observación y sentido para interpretar los cambios que muestra el potro, actuando de manera acertada para lograr el objetivo propuesto (Hoyos 2009b).

Continuando con el proceso de manejo y manipulación del potro, se encuentra el descosquillado, dentro de este concepto están inmersas infinidad de actividades, las cuales buscan un potro dócil que se deje tocar todo el cuerpo, entregue patas y manos, acepte todos los aperos y arreos sin oponer resistencia y esté listo para que el humano lo monte (Hoyos, 2009c).

En los sistemas de doma usados actualmente se puede abordar esta fase desde dos puntos de vista, el primero es amarrar el potro a un botalón, ensueltarlo (sujetarlo de manos y patas) y comenzar a acariciarlo sin ganar su confianza; este sistema pone al potro en un estado máximo de alerta activando el sistema de defensa, invadiendo la zona de protección de éste. El segundo sistema, busca la aceptación por parte del potro y el acercamiento progresivo del humano al espacio vital de éste, para entablar una relación de seguridad y confianza, donde el hombre toma el papel de $\alpha$ (alfa) de y el potro acepta su puesto en la manada (Roberts, 2004; Hoyos, 2009d).

Ya en este punto del proceso el domador ha notado los puntos favorables y desfavorables que presenta el potro con respecto a la ejecución de los ejercicios, y la forma como atiende las órdenes; en muchos casos las deficiencias no son notorias para el domador por su sutileza, se requiere de ayudas, tales como videos o ayudantes, para analizar detenidamente el conjunto del caballo (performance), para corregir las causas y no las consecuencias, que dan origen a las irregularidades (Hoyos, 2010a).

Con referencia en lo que se ha dado en denominar psicología del caballo, se pueden diferenciar 2 grandes etapas en la relación hombre-caballo: la doma y el adiestramiento, la primera dividida en dos 
fases la domesticación y el aprendizaje y la segunda comprende dentro del sistema de doma para el Caballo Criollo Colombiano, la puesta a punto en el freno y la reunión del caballo (Hoyos, 2010a).

Dentro del picadero se desarrolla el trabajo a la cuerda, este no se puede limitar a una serie de vueltas en el torno sin ninguna finalidad, buscara equilibrar al potro y mostrarle al domador sus características, permitiendo enfocar el trabajo a desarrollar. Para el trabajo a la cuerda, es conveniente detenerse y observar ciertos puntos importantes, en conformación y desplazamiento del potro, contestando las siguientes interrogantes: ¿Inserción del cuello con relación a encuentro y pecho?; ¿inserción cuello-cabeza?; ¿Cómo se equilibra el potro al realizar el paso, trote y aire natural de desplazamiento?; ¿Estas marcas son rítmicas?; ¿La línea del lomo se hunde, es rígido o es fuerte? Al diagnosticar estos aspectos se reúnen las bases necesarias para ejecutar un trabajo a la cuerda que ayude a corregir, desarrollar o mejorar las características que presenta el potro (Hoyos, 2011a).

En el esquema de doma tradicional, el trabajo se desarrolla desde el lomo del caballo y no se prepara a éste para soportar el peso del jinete; no se tiene en cuenta la fuerza ejercida en el lomo del potro al momento de montarlo, generando infinidad de problemas cuando se quiere reunir el caballo y se busca que el tren posterior trabaje correctamente como motor en el desplazamiento (Hoyos, 2008e; Quiroz, et al, 2015b). El equilibrio se pierde cuando el potro soporta peso sobre el dorso; al sostener peso, contrae y tensa los músculos dorsales, en especial el gran dorsal; que no está diseñado para soportar el peso del jinete y mantener la tensión ejercida a lo largo de la columna vertebral, provocando cansancio y dolor después del trabajo (Hempfling, 1996; Hoyos 2008e).

El potro no puede mantener la tensión en el dorso y lo relaja, llevando el peso del chalan sobre los huesos, sin soporte y amortiguación muscular, resultando en el hundimiento del lomo y pérdida de elasticidad en posteriores, fallando la locomoción, caracterizada por desplazamiento en miembros en arrastre, perdiendo impulsión y elevación (Hoyos, 2009a). Al montarlo, éste se equilibra con el peso de cabeza y cuello actuando como contrapeso (Figura 3 y 4); al comienzo como los músculos de la parte superior del cuello no están desarrollados es de resaltar que a mayor peso del jinete, más abajo debe llevar la cabeza el potro, con esta medida se busca el efecto de contrapeso de la cabeza y el
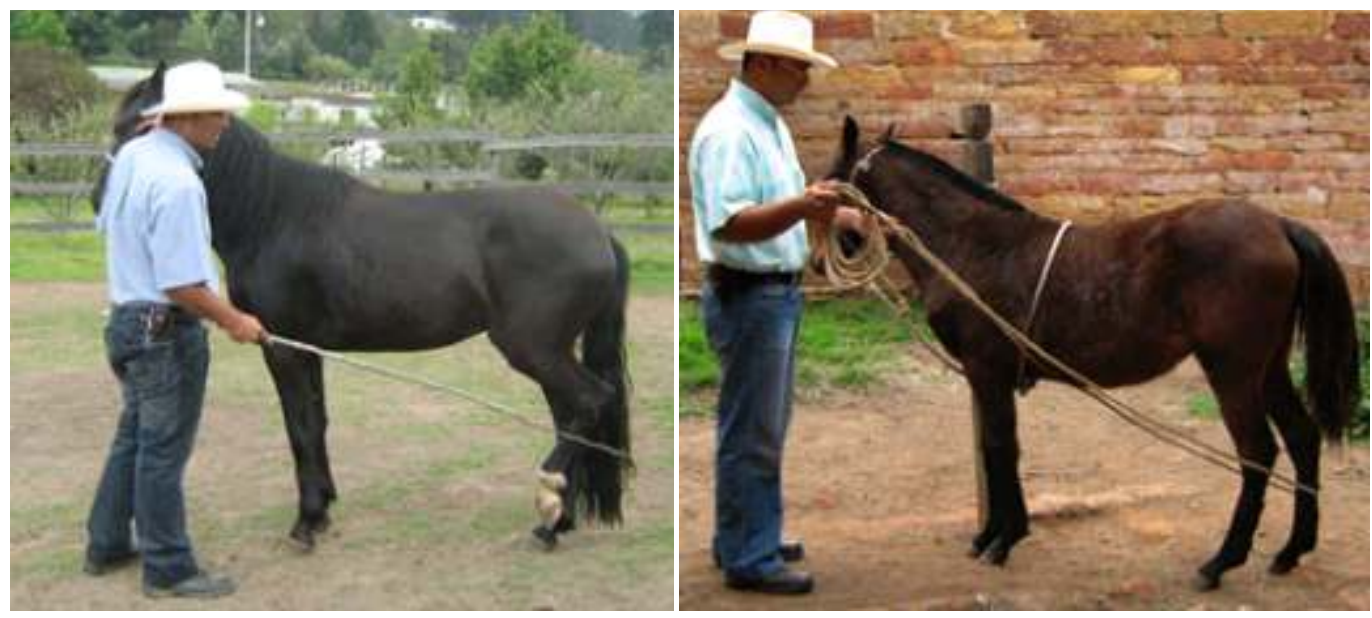

Figura 3. (Izq) y 4 (Der): método de descosquillado extremidades posteriores. Figura 3 Potro dentro del picadero y uso de vara; Figura 4 Potro sujeto al botalón uso de cuerda o lazo. Fuente: Revista Universo Equinos $\mathrm{N}^{\circ} 7$. 


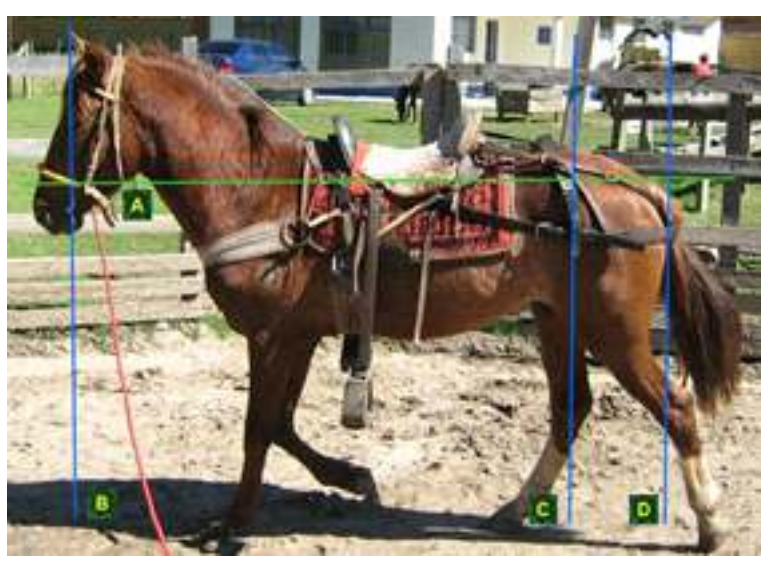

Figura 5. ANTES

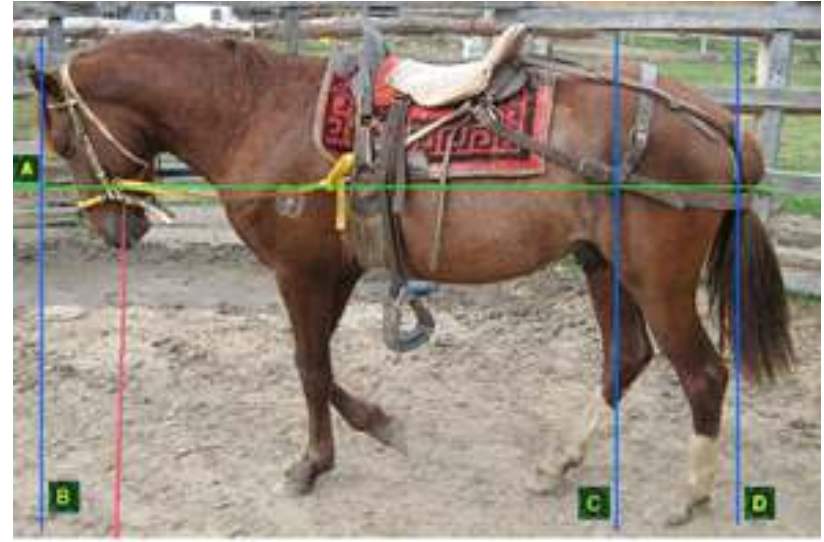

Figura 6. DESPUÉS

Líneas imaginarias efecto trabajo lomo del potro (Trabajo rienda corta)

Figura 5. Caballo desplazándose en equilibrio natural:

A. La línea horizontal que pasa por la argolla del bozal esta desplazada hacia arriba con respecto a la línea media del caballo.

B. La cara del caballo esta por fuera del ángulo recto que se debe formar con respecto al suelo.

C y D. Línea vertical que muestra el desplazamiento de la pata hacia fuera del punto de impulsión del movimiento.

Figura 6. Caballo desplazándose con el uso de rienda corta, preparado para llevar peso sobre su lomo: (nótese la incurvación del lomo y el desplazamiento del anca y tren posterior hacia adentro y por debajo de la masa del cuerpo).

A. A. Línea horizontal formada entre la argolla del bozal y la argolla de la montura (3), rienda fija sin tensión.

B. B. Línea vertical que forma el ángulo de trabajo con la cara del potro, flexión del cuello trabajo pasivo de la cabeza.

C y D. Línea vertical que indica el trabajo del tren posterior, llevando la pata por debajo del centro de equilibrio, creando la impulsión del movimiento (2). Fuente: Revista Universo Equino Nº.

cuello activando el ligamento supraespinoso liberando así, los músculos dorsales y los músculos de la grupa brindando una locomoción óptima, un dorso relajado y elástico (Hempfling, 1996; Hoyos 2009a).

Por otro lado, la flexión del cuello o quebrantada ocupa un reglón primordial en el proceso de doma tradicional, este se efectúa de innumerables maneras, todas buscando un mismo fin, que el potro afloje el conjunto cabeza-cuello (articulación atlanto-occipital y zona de la tabla del cuello) y sea más dócil a la mano (Hoyos, 2011b). El problema se presenta cuando se aplica excesiva fuerza para lograrlo o se realizan series interminables de repeticiones del ejercicio, obteniendo así, animales que entregan al menor toque la cabeza, para evitar molestia, perdiendo el objetivo de mejorar la flexibilidad para el trabajo (Hoyos, 2013).

Continuando con el trabajo dentro del picadero, el siguiente paso es buscar ensillar el potro. Primero se le permite olfatear todas las herramientas con las que se trabaja; se le presenta la alfombra o sudadero y se frota todo el cuerpo teniendo cuidado de hacerlo por ambos lados (Hoyos, 2009d). Seguidamente, se presenta la montura y se realiza el mismo proceso teniendo cuidado de ponerla sobre el lomo y premiar al potro; posteriormente, se retira para hacerle entender que la acción es 
temporal; acto seguido se vuelve a poner la silla en el lomo y se cincha, ajustan los colgantes extras como la baticola y pechera, moviendo al potro primero cabestreado, después al paso y luego al trote (Hoyos, 2010b).

Cuando el potro trabaja correctamente en el picadero permitiendo ser montado, flexionando el cuello con facilidad, atendiendo a la orden de alto y retroceso, se inicia el trabajo en giros, cambios de marcha dentro y fuera del picadero, para obtener que se desplace sin problemas con el jinete sobre el lomo (Hoyos, 2010c).

Todos los trabajos se deben iniciar al paso para que el caballo los entienda y pueda asimilarlos de la mejor manera; en esta parte del trabajo el potro se encontrará con diferentes ayudas con las que el domador le va hacer entender sus demandas, estas ayudas consisten en los apoyos de la rienda, el cuerpo y la pierna para hacerle girar fácilmente sin que éste pierda la impulsión del tren posterior ni saque los posteriores (Hoyos, 2010c). Primero que todo se debe recordar que el caballo evita la presión, esto quiere decir que al momento de sentir un estímulo se aleja de él; este concepto se debe tener en cuenta, al momento de enseñarlo a girar y pararlo (Hoyos, 2010a).

\section{CONCLUSIONES}

El análisis de la presente revisión documental, reúne algunos aspectos relevantes sobre las nuevas técnicas de doma usadas para el caballo criollo colombiano, permitiendo llegar a las siguientes conclusiones:

La implementación del Imprinting como técnica rutinaria en los criaderos mejora el manejo y evita la presentación de posteriores vicios. La doma de pesebrera como paso siguiente en la habitación del potro, permite que el potro desteto reafirme la confianza con el humano y su entorno.

El uso del picadero como herramienta para el inicio del potro, brinda seguridad para el binomio de trabajo, crea una ambiente de atención optimizando los resultados. La preparación física del potro antes de la primera montada es fundamental para evitar futuras lesiones y propender por una vida productiva larga y sin lesiones, fortaleciendo la musculatura del lomo y el trabajo en impulsión.

Tanto la doma tradicional como la etológica, son exitosas para el adiestramiento del caballo; sin embrago, en tradicional, los potros se muestran temerosos y excitables, desconfiados al contacto con el humano. La aplicación de las doma etológicas o racional, permite que el potro se exprese naturalmente, mostrando confianza con el domador y el entorno. Permitiendo así, la expresión de todo el potencial genético del equino.

\section{LITERATURA CITADA}

CALMO, J. A. F. D. Gente que ama cavalos: a compreensão das práticas equestres como objeto de estudo antropológico a partir da vivência na Escola de Equitação Cristal em Porto Alegre-RS. 2015. \{En línea\}. Disponible en: https://cutt.ly/2yH1Cx5

CORREDOR, L. Caracterización anatomofisiológica y estudio comportamental del caballo de monta para equinoterapia. Universidad de la Salle- Programa de medicina Veterinaria-Facultad de Ciencias 
agropecuarias. 2009. \{En línea\}. Disponible en: https://cutt.ly/jyHXJS3

COSTA, B. O., FERREIRA, T. M. V., CAROLINE, S., SOUZA, B., \& NUNES-PINHEIRO, D. C. S. Efeito do treinamento de doma sobre os parâmetros físicos e hematobioquímicos de equinos, antes e após o exercício. Ci.Anim. 2016. Pp. 35-50.\{En línea\}. Disponible en: https://cutt.ly/syJD2LY

DE SOUZA, A., DE SOUZA JÚNIOR, J. B., DE SOUZA, E. M., DOS REIS FARIA, M., \& MOURO, G. F. 13885-Bem-estar animal e doma de equinos: um estudo em Lunardelli-PR. Cadernos de Agroecologia, 8(2). 2013. \{En línea\}. Disponible en: https://cutt.ly/JyHXL3w

ESTRADA, R. Chalanería Colombiana II y otros temas. [ed.] Raúl Estrada Londoño Medellín: Vieco y Cia.1994

ESTRADA, R. Chalanería Colombiana. [ed.] Raúl Estrada Londoño. Segunda edición. Medellín: Vieco y Cia. 1991

HEMPFLING, K F. Tratar con caballos: estudio de la comunicación real con el caballo; intimidad y armonía desde el principio. Guías del naturalista-animales domésticos-caballos. Editor Omega. ISBN 8428210616, 9788428210614. 1996

HERNÁNDEZ, F M A. ¿Cómo el estudio de la biomecánica en el caballo criollo puede influir en su vida competitiva? Colegio Marymount. Proyecto de Grado. Medellín. 2015. \{En línea\}. Disponible en: https://cutt.ly/EyJIMbn

HOYOS PATIÑO, J. F., \& HERNANDEZ VILLAMIZAR, D. A. MANUAL DE ESTIMULACION EN POTROS IMPRINTING. 2020. \{En línea\}. Disponible en: https://cutt.ly/dyJDU6g

HOYOS-PATIÑO, J. F Doma de Pesebrera. Segundo pasó en la habitación de los potros. Revista El Caballo. Vol 12.Pp. 26-28. ISSN. 1900-348X. 2007d. \{En línea\}. Disponible en: https://cutt.ly/SyJLQUm

HOYOS-PATIÑO, J. F. Acostumbramiento temprano. Revista Acontecer Equino. Boletín coleccionable ASDEPASO. vol 12.2007c. \{En línea\}. Disponible en: https://cutt.ly/5yJGRXR

HOYOS-PATIÑO, J. F. Adiestramiento etología del caballo. Revista Universo Equino. Vol. 2. Pp 71-74. ISSN 2665-5551. Pereira. Colombia. 2008a . \{En línea\}. Disponible en: https://cutt.ly/LyJD3jb

HOYOS-PATIÑO, J. F. Doma de pesebrera. (Habituación de los potros antes de los 18 meses). Revista Universo Equino. Vol. 3. ISSN 2665-5551. Pereira. Colombia. 2008b. Pp 71-74. \{En línea\}. Disponible en: https://cutt.ly/6yJLvu5

HOYOS-PATIÑO, J. F. Doma de Pesebrera. Segundo pasó en la habitación de los potros II. Revista El Caballo. Vol 13. Pp. 60-62. ISSN. 1900-348X.2008c. \{En línea\}. Disponible en: https://cutt.ly/5yJLRMM

HOYOS-PATIÑO, J. F. El lomo del caballo (caballos pandos), segunda parte. Revista Universo Equino $\mathrm{N}^{\circ} 6$ marzo-abril. ISSN 2665-5551.Pereira. Colombia. 2009a . En línea $\}$. Disponible en: https://cutt.ly/lyJD6nx

HOYOS-PATIÑO, J. F. Estimulación Temprana en potros (Imprinting). Revista El Caballo. Vol 11. Pp. 38-40. ISSN. 1900-348X. 2007a. \{En línea\}. Disponible en: https://cutt.ly/YyJDZLD 
HOYOS-PATIÑO, J. F. Evaluación comparativa de la aplicación del imprinting en potros criollos colombianos. FAGROPEC-Facultad de Ciencias Agropecuarias, 8(2), 2016. Pp. 62-67. \{En línea\}. Disponible en: https://cutt.ly/9yJGLTN

HOYOS-PATIÑO, J. F. Evaluación de una guía metodológica para la explicación de la enseñanza temprana Imprinting en potros recién nacidos criollos Colombianos. Trabajo de grado presentado como requisito parcial para optar el título de Zootecnista. Tutor: Daniel Antonio Hernández Villamizar. Facultad de ciencias agrarias. Universidad de Pamplona. Norte de Santander. 2006

HOYOS-PATIÑO, J. F. Experto Colombiano explica las fases del Imprinting. Revista Colombia Equina. Vol 4. 2007b. Pp.142-148. \{En línea\}. Disponible en: https://cutt.ly/XyJGnf6

HOYOS-PATIÑO, J. F. Terapia de extensión y flexión para el equino. Parte I. Revista Universo Equino. Vol. 22. Pp 8-10-12-14. ISSN 2665-5551. Pereira. Colombia. 2013. \{En línea \}. Disponible en: https://cutt.ly/zyJL92e

HOYOS-PATIÑO, J. F., BERMÚDEZ-GUTIÉRREZ, E., HERNÁNDEZ-VILLAMIZAR, D. A., \& VELÁSQUEZ-CARRASCAL, B. L. Aplicación del protocolo Welfare Quality ${ }^{\circledR}$ en criaderos equinos para determinar el grado de bienestar animal. Mundo FESC, 9(18), 24-30. 2019. \{En línea\}.Disponible en: https://cutt.ly/5yJOWN1

HOYOS-PATIÑO, J. F., SUAREZ SALAZAR, J. C., \& ESTRADA CELY, G. E. Evaluación comparativa de un método de doma humanitaria y uno tradicional para el caballo de silla Colombiano. Revista Respuestas. Universidad Francisco de Paula Santander. 2020

HOYOS-PATIÑO, J. F.Bases de rienda. Trabajo del potro fuera del picadero. Revista Universo Equino. Vol. 12. Pp 12-14-16. ISSN 2665-5551. Pereira. Colombia.2010c. \{En línea\}. Disponible en: https://cutt.ly/ByJL5Kv

HOYOS-PATIÑO, J. F.Conocimiento del potro que se va a domar. Revista Universo Equino. Vol. 10. Pp 1012-14-16-58-59. ISSN 2665-5551. Pereira. Colombia.2009b. \{En línea\}. Disponible en: https://cutt.ly/lyJLY9E

HOYOS-PATIÑO, J. F.Efecto de la relajación y flexibilidad del conjunto cabeza-cuello en el desempeño del equino. Revista Universo Equino. Vol. 15. Pp 12-14-15-16. ISSN 2665-5551. Pereira. Colombia. 2011 b. $\{$ En línea\}.Disponible en: https://cutt.ly/0yJL0Fb

HOYOS-PATIÑO, J. F.El cabezal de nudos. Revista Universo Equino. Vol. 4. Pp 74-77. ISSN 2665-5551. Pereira. Colombia. 2008d. \{En línea\}. Disponible en: https://cutt.ly/lyJLY9E

HOYOS-PATIÑO, J. F.El descosquillado. Un proceso fundamental en la doma (Primera Parte). Revista Universo Equino. Vol. 7. Pp 82-95. ISSN 2665-5551. Pereira. Colombia. 2009c. \{En línea\}. Disponible en: https://cutt.ly/nyJLGvx

HOYOS-PATIÑO, J. F.El descosquillado. Un proceso fundamental en la doma (Segunda Parte). Revista Universo Equino. Vol. 8. Pp 8-10-12-14. ISSN 2665-5551. Pereira. Colombia. 2009d.\{En línea\}. Disponible en: https://cutt.ly/lyJLLcL

HOYOS-PATIÑO, J. F.El lomo del caballo (caballos pandos), primera parte. Revista Universo Equino vol. 5 
pp. 68-70-71 ISSN 2665-5551.Pereira. Colombia. 2008e. \{En línea $\}$. Disponible en: https://cutt.ly/QyJLB7z

HOYOS-PATIÑO, J. F.La primera montada. Revista Universo Equino. Vol. 11. Pp 44-46-48-50. ISSN 26655551. Pereira. Colombia. 2010b. \{En línea\}. Disponible en: https://cutt.ly/FyJL4vR

HOYOS-PATIÑO, J. F.Proceso de aprendizaje del potro. Revista Universo Equino. Vol. 13. Pp 44-46-48-50. ISSN 2665-5551. Pereira. Colombia.2010a . \{En línea . Disponible en: https://cutt.ly/ayJLXBO

HOYOS-PATIÑO, J. F.Trabajo a la cuerda. Revista Universo Equino. Vol. 16. Pp 24-26-28-30-34-36 ISSN 2665-5551.Pereira. Colombia. 2011 a . \{En línea $\}$.Disponible en: https://cutt.ly/TyJZrem

HOYOS-PATIÑO, J. F., \& FRANCO-R, E. La cría del caballo de paso en Norte de Santander. Revista Universo Equino. Vol.29.ISSN 2665-555. Pereira. Colombia. 2017. Pp 12,14,16. \{En línea\}. Disponible en: https://cutt.ly/DyJI41k

LEÓN, RA L., \& CONTRERAS G K M. Estudio morfológico del caballo de monta para equinoterapia (tesis de pregrado). Director: Johann Fernando Hoyos Patiño. Facultad de ciencias agrarias. Universidad de Pamplona. Colombia. 2016

MARTÍNEZ, S J J. Caracterización de los procesos de doma aplicados al caballo criollo colombiano. (Tesis de pregrado). Director: Johann Fernando Hoyos Patiño. Facultad de ciencias agrarias. Universidad de Pamplona. Colombia.2016

MARTOS, N., \& AYALA, I. El estrés en los equinos. Anales de veterinaria de Murcia.19. 2003.Pp.121,127. Disponible en: https://cutt.ly/CyJF3lb

OLIVEIRA, J. N., \& FERGITZ, A. C. Manejo enquanto potro visando o bem-estar enquanto cavalo atleta: Revisão Bibliográfica. 2018. \{En línea\}. Disponible en: https://cutt.ly/ryJL17f

OLIVEIRA, J. N., MACHADO, R. B., \& PEREIRA, A. T. Imprinting training e manejo de potros do nascimento até a desmama, visando ao bem-estar animal, na coudelaria da serra-1 regimento de polícia montada da brigada militar-rs. 2016. \{En línea\}. Disponible en: https://cutt.ly/CyJGHyg

PATIÑO, J. F. H., \& GÓMEZ, R. A. CARACTERIZACIÓN DE LAS TENDENCIAS EN LA DOMA DE CABALLOS DE SILLA COLOMBIANO. FAGROPEC-Facultad de Ciencias Agropecuarias, 8(1). 2016. \{En línea\}. Disponible en: https://cutt.ly/jyJDjfE

PISA, J. P. N., TACITO, J. L. C., \& LEME, D. P. Relação humano-equino a partir da obra. PUBVET, 13, 158. 2019. \{En línea\}. Disponible en: https://cutt.ly/FyJOlAz

QUIROZ, B. E. P., ROMERO, N. E. B., \& ORTÍZ, C. A. Z. Diagnóstico y descripción de conductas estereotipadas en equinos bajo condiciones de pesebrera en Florencia-Caquetá. FAGROPEC-Facultad de Ciencias Agropecuarias, 7(1).2015 . \{En línea\}. Disponible en: https://cutt.ly/7yJLOUX

QUIROZ, B. E. P., ROMERO, N. E. B., SABI, C. H., \& DUSSAN, R. C. Caracterización morfométrica en equinos utilizados como herramienta de tracción en florencia-caquetá. FAGROPEC-Facultad de Ciencias Agropecuarias, 7(1).2015b. \{En línea\}. Disponible en: https://cutt.ly/XyJZueP 
REINOS, J. A. C., \& PATIÑO, J. F. H. COMPARACIÓN DEL EFECTO DEL PROCESO DE DOMA HUMANITARIA EN POTROS IMPRONTADOS. FAGROPEC-Facultad de Ciencias Agropecuarias, 9(1), 2017. Pp. 5-10. .\{En línea $\}$. Disponible en: https://cutt.ly/LyJG0sz

ROBERTS, M. De mis manos a las tuyas. Solvang, CA: Editorial Tutor, 2004.

SANNA, T R. Estudio de factibilidad para la implementación de un centro de equinoterapia de la Universidad de Pamplona (tesis de pregrado). Directora: Blanca Liliana Velázquez Carrascal. Facultad de ciencias agrarias. Universidad de Pamplona. Colombia. 2017

SANTOS, V. H. D. S. Doma racional e treinamento de rédeas (Bachelor's thesis, Brasil). 2019.\{En línea\}.Disponible en: https://cutt.ly/nyJDbuP

VIEIRA, M. C. Percepções de práticas de manejo em estabelecimentos equestres quanto à influência dessas práticas para o bem-estar de equinos. 2015.\{En línea\}. Disponible en: https://cutt.ly/hyJD7V0 